\title{
Cubature Error Bounds for Analytic Functions
}

\author{
By F. G. Lether
}

\begin{abstract}
In this paper, two-dimensional cubature error bounds are developed. It is assumed that the function to be integrated is analytic, and that the domain of integration is contained in $[-1,1] \times[-1,1]$.

Tables of error constants for several cubature rules and domains of integration are included.
\end{abstract}

1. Introduction. The purpose of this paper is to develop cubature error bounds for a general class of rules. The weight function is not required to be unity and the region of integration need not be a square. It is assumed that the function $f$, to be integrated, is jointly analytic in each variable inside a disc of radius $r>1$. We employ a technique similar to that used by Stenger [17], and apply the cubature error functional to the double Taylor series for $f$. This analysis has the advantage of being elementary and leads to derivative-free error bounds.

Many of the cubature error results which have appeared in the literature are restricted to cross-product rules. We mention the work of the following authors: [1], [8], [13], [18]. In general, these bounds cannot be easily extended to nonproduct rules.

It is possible to use the kernel theorems of Sard [14] to obtain error bounds for nonproduct rules. However, these bounds involve partial derivatives of $f$, which are often difficult to work with. In using Sard's results, as given in [19, Chapter 5] one must decide which of the many possible spaces $B_{p, a}, B_{|p, a|}$ to work in. It may be necessary to take several values of $p$ and $q$ to find an acceptable error bound. Stroud [19, Chapter 8] has recently computed the Sard error constants for several cubature rules. Most of these constants are for rules over $[-1,1] \times[-1,1]$. They are difficult to compute, and impractical to tabulate for moderately high order rules in 3 or more dimensions.

Barnhill [2] has used Hilbert space techniques to develop derivative-free error bounds. His results are not restricted to cross-product rules and the domain of integration need not be a square. We remark that Chawla [4] has also obtained derivative-free cubature error bounds through Cauchy's formula. Chawla's error bounds are similar to Barnhill's. The error constants, for both of these error bounds and the bounds developed in this paper, depend on the region of regularity of $f$. It is therefore necessary to tabulate several error constants for a fixed cubature rule. Stroud [19] has recently tabulated the Barnhill error constants for several rules. However, the error constants necessary for the practical application of Chawla's error bound have not been tabulated.

\footnotetext{
Received February 18, 1972, revised May 12, 1972.

AMS (MOS) subject classifications (1970). Primary 65D30; Secondary 65D20, 30A10. quality.

Key words and phrases. Cubature error bounds, Taylor series, closed bicylinder, Cauchy ine-
} 
In Section 2 of this paper, we give an elementary derivation of an error bound for a general cubature rule in two dimensions. Several numerical examples are given which illustrate the application of the theory. The error bound obtained in this paper is compared with the error bounds of Barnhill and Chawla in Section 3. The error constants needed for our bound are tabulated in Section 4 for several of the rules which appear in [19].

Although we treat only the two-dimensional case here, the extension of our results to higher dimensions will be obvious.

2. Error Bounds. Let the cubature rule

$$
\int_{D} \int \omega(x, y) f(x, y) d y d x=\sum_{k=1}^{N} w_{k} f\left(x_{k}, y_{k}\right)+E(f)
$$

have precision $p$. We require $\left(x_{k}, y_{k}\right) \in D, 1 \leqq k \leqq N$ and $D \subseteq S$, where $S=[-1,1]$ $\times[-1,1]$.

In the following work, $z=x+i u$ and $w=y+i v$, where $x, y, u$ and $v$ are real. Let $B_{r}$ denote the closed bicylinder of radius $r>1$, defined by $\{(z, w):|z| \leqq r$, $|w| \leqq r\}$. The class of all functions $f(z, w)$ that are real when $z$ and $w$ are real and such that $f(z, w)$ is analytic in $B_{r}$ is designated by $A\left(B_{r}\right)$.

THEOREM 1. Let $f \in A\left(B_{r}\right), r>1$. Then

$$
|E(f)| \leqq e_{r} M(r),
$$

where

$$
e_{r}=\sum_{\mu+\nu \geqq p+1} r^{-\mu-\nu}\left|E\left(x^{\mu} y^{\nu}\right)\right|
$$

and

$$
M(r)=\max _{|z|=|w|=r}|f(z, w)| .
$$

If the errors $E\left(x^{\mu} y^{\nu}\right), \mu+\nu \geqq p+1$, have the same sign, then

$$
e_{r}=\left|E\left[(1-x / r)^{-1}(1-y / r)^{-1}\right]\right| .
$$

Proof. $f \in A\left(B_{r}\right)$ implies that, at all points $(z, w) \in B_{r}$,

$$
f(z, w)=\sum_{\mu+\nu \geq 0} a_{\mu \nu} z^{\mu} w^{\nu},
$$

where

$$
\mu ! \nu ! a_{\mu \nu}=\left.\frac{\partial^{\mu+\nu} f(z, w)}{\partial z^{\mu} \partial w^{\nu}}\right|_{z=w=0} .
$$

This double Taylor series converges absolutely and uniformly in the closed bicylinder $B_{r}$ and Cauchy's inequality [5] holds:

$$
\left|a_{\mu \nu}\right| \leqq r^{-\mu-\nu} M(r) .
$$

$r>1$ implies $D \subseteq S \subseteq B_{r}$. Thus, 


$$
E(f)=\sum_{\mu+\nu \geq p+1} a_{\mu \nu} E\left(x^{\mu} y^{\nu}\right)
$$

and

$$
\begin{aligned}
|E(f)| & \leqq \sum_{\mu+\nu \geq p+1}\left|a_{\mu \nu}\right|\left|E\left(x^{\mu} y^{\nu}\right)\right| \\
& \leqq M(r) \sum_{\mu+\nu \geq p+1} r^{-\mu-\nu}\left|E\left(x^{\mu} y^{\nu}\right)\right|=e_{r} M(r) .
\end{aligned}
$$

Let

$$
h(x, y)=(1-x / r)^{-1}(1-y / r)^{-1} .
$$

The Taylor series

$$
h(x, y)=\sum_{\mu+\nu \geq 0} r^{-\mu-\gamma} x^{\mu} y^{\nu}
$$

converges uniformly on $D$. Consequently,

$$
E(h)=\sum_{\mu+v \geq p+1} r^{-\mu-v} E\left(x^{\mu} y^{\nu}\right) .
$$

Suppose $E\left(x^{\mu} y^{\nu}\right) \geqq 0, \mu+\nu \geqq p+1$. Then, $e_{r}=E(h)$. Similarly, $e_{r}=-E(h)$ if $E\left(x^{\mu} y^{\nu}\right) \leqq 0, \mu+\nu \geqq p+1$. It follows that $e_{r}=|E(h)|$, provided the errors $E\left(x^{\mu} y^{\nu}\right)$, $\mu+\nu \geqq p+1$, have the same sign. This completes the proof.

In the error bound (2.2), it is necessary to evaluate $M(r)$. This involves computing the maximum modulus of $f(z, w)$ on the distinguished boundary $\{(z, w):|z|=r$, $|w|=r\}$ of $B_{r}$. If the Taylor coefficients $a_{\mu \nu}$ have the same sign for $\mu+\nu \geqq 0$, then $M(r)=|f(r, r)|$. This condition on the Taylor coefficients will sometimes be evident without our having explicit values for the $a_{\mu \nu}$, for example, if $f(x, y)=$ $(20-x-y)^{-2} \tan (x / 6)$. It may also be possible to compute $M(r)$ by evaluating $|f(z, w)|$ at a sufficiently dense set of points on the distinguished boundary of $B_{r}$. It should be noted that the error bounds in [2] and [4] also require the computation of a quantity analogous to $M(r)$.

The error constant $e_{r}$ depends on $r$. It is therefore necessary to tabulate $e_{r}$ for several values of $r$, for a fixed cubature rule. If $f \in A\left(B_{r}\right), 1<r<R \leqq \infty$, then

$$
|E(f)| \leqq \inf _{1<r<R} e_{r} M(r) .
$$

Example 1. The cubature rule

$$
\begin{aligned}
\int_{0}^{1} \int_{0}^{1-x} f(x, y) d y d x= & \frac{1}{60}\left[f\left(\frac{1}{2}, \frac{1}{2}\right)+f\left(\frac{1}{2}, 0\right)+f\left(0, \frac{1}{2}\right)\right] \\
& +\frac{9}{60}\left[f\left(\frac{1}{6}, \frac{1}{6}\right)+f\left(\frac{1}{6}, \frac{2}{3}\right)+f\left(\frac{2}{3}, \frac{1}{6}\right)\right]+E(f)
\end{aligned}
$$

has precision $p=3$. Suppose this rule is used to approximate the integral

$$
\int_{0}^{1} \int_{0}^{1-x} \frac{\tan (x / 6)}{(20-x-y)^{2}} d y d x=.7519481768(-4)
$$

where the value in parentheses indicates the power of 10 by which the preceding number should be multiplied.

The integrand is in $A\left(B_{r}\right)$ for $1<r<3 \pi$. By our above remarks and (2.2), 


$$
|E| \leqq e_{r}(20-2 r)^{-2} \tan (r / 6)
$$

The right side of this inequality is minimized when $r=6$ in Table 1. For this value of $r$, we obtain $|E| \leqq .1230(-6)$. The true error is $|E|=.6430(-9)$.

The error bound (2.2) can be improved if (2.1) is a fully symmetric rule [7]. Consider the function $g$ defined by

$$
g(z, w)=\frac{1}{4}[f( \pm z, \pm w)]=\sum_{\mu+\nu \geqq 0}^{*} a_{\mu \nu} z^{\mu} w^{\nu}
$$

where the asterisk indicates that the sum is to be taken over the even indices only, and

$$
f( \pm z, \pm w)=f(z, w)+f(-z, w)+f(-z,-w)+f(z,-w) .
$$

Set

$$
N(r)=\max _{|z|=|w|=r}|g(z, w)| .
$$

Clearly, $N(r) \leqq M(r)$ and, by Cauchy's inequality,

$$
\left|a_{\mu \nu}\right| \leqq r^{-\mu-\nu} N(r) \text {, }
$$

for even $\mu$ and $\nu$. If (2.1) is fully symmetric, then $E(f)=E(g)$. It follows that (2.2) is valid if we replace $M(r)$ by $N(r)$. Even though $g$ will usually be more complicated than $f$, there may be instances where it is worthwhile to use $N(r)$. If the Taylor coefficients of $f$, with even subscripts, are all of the same sign, then $N(r)=|g(r, r)|$. The function $f(x, y)=(x+y+16)^{-1}$ has these properties and for it $N(r)=32^{-1}+$ $2\left(64-r^{2}\right)^{-1}$, in contrast to $M(r)=(16-2 r)^{-1}$.

Example 2. Consider the fully symmetric cubature rule

$$
\iint_{x^{2}+y^{2} \leqq 1} f(x, y) d y d x=\frac{\pi}{8}\left[f\left( \pm \frac{2^{1 / 2}}{2}, 0\right)+f\left(0, \pm \frac{2^{1 / 2}}{2}\right)\right]+E(f),
$$

which has precision $p=3$. We use this rule to approximate

$$
\iint_{x^{2}+\nu^{2} \leqq 1} \frac{d y d x}{x+y+16}=.1967345414 .
$$

TABLE 1

Error Constants for Example 1

\begin{tabular}{cc}
\hline$r$ & $e_{r}$ \\
\hline 1.1 & $.5360(-1)$ \\
1.2 & $.2362(-1)$ \\
1.3 & $.1251(-1)$ \\
1.4 & $.7371(-2)$ \\
1.6 & $.3122(-2)$ \\
2.0 & $.8755(-3)$ \\
3.0 & $.1142(-3)$ \\
4.0 & $.3020(-4)$ \\
6.0 & $.5053(-5)$ \\
8.0 & $.1478(-5)$ \\
\hline
\end{tabular}


The error bound (2.2), with $M(r)$ replaced by $N(r)$, gives

$$
|E| \leqq e_{r}\left(1 / 32+2 /\left(64-r^{2}\right)\right) \text {. }
$$

Taking $r=6$ in Table 2, we obtain $|E| \leqq .1081(-4)$. The actual error is $|E|=$ $.7549(-6)$.

In certain cases, $(2.4)$ is a convenient alternative to (2.3) for the computation of $e_{r}$. Suppose $\omega(x, y) \equiv 1$ and $D=[-1,1] \times[-1,1]$ in (2.1). Then, (2.1) and (2.4) give

$$
e_{r}=\left|\left(r \ln \frac{r+1}{r-1}\right)^{2}-\sum_{k=1}^{N} w_{k}\left(1-\frac{x_{k}}{r}\right)^{-1}\left(1-\frac{y_{k}}{r}\right)^{-1}\right| .
$$

If the errors $E\left(x^{\mu} y^{\nu}\right), \mu+\nu \geqq p+1$, have the same sign, then (2.5) is preferable to (2.3). In particular, the error representation given in [9] can easily be used to show that $E\left(x^{\mu} y^{\nu}\right) \geqq 0$ when $(2.1)$ is a Gauss-Legendre product rule.

It is possible to bound $e_{r}$. Let

$$
c=\sup _{\mu+\nu \geq p+1}\left|E\left(x^{\mu} y^{\nu}\right)\right| \text { and } \delta(r)=\sum_{\mu+\nu \geq p+1}^{\prime} r^{-\mu-\nu},
$$

where the prime on the summation indicates that the sum is to be taken over the indices $\mu+\nu \geqq p+1$ for which $E\left(x^{\mu} y^{\nu}\right) \neq 0$. Clearly, $c<\infty$. By the proof of Theorem 1 and (2.3),

$$
\left|E\left[(1-x / r)^{-1}(1-y / r)^{-1}\right]\right| \leqq e_{r}<c \delta(r) .
$$

We have

$$
\delta(r) \leqq \sum_{\mu+\nu \geq p+1} r^{-\mu-\nu}=\frac{(p+2) r-(p+1)}{r^{p}(r-1)^{2}} .
$$

For certain classes of cubature rules, a closed form for $\delta(r)$ can be calculated. If (2.1) is a fully symmetric rule, then

$$
\delta(r)=\frac{(p+3) r^{2}-(p+1)}{2 r^{p-1}\left(r^{2}-1\right)^{2}} .
$$

TABLE 2

Error Constants for Example 2

\begin{tabular}{ll}
\hline$r$ & \multicolumn{1}{c}{$e_{r}$} \\
\hline 1.1 & .5862 \\
1.2 & .2619 \\
1.3 & .1430 \\
1.4 & $.8712(-1)$ \\
1.6 & $.3939(-1)$ \\
2.0 & $.1232(-1)$ \\
3.0 & $.1921(-2)$ \\
4.0 & $.5629(-3)$ \\
6.0 & $.1053(-3)$ \\
8.0 & $.3272(-4)$ \\
\hline
\end{tabular}


TABLE 3

Error Constants for Example 3

\begin{tabular}{ccc}
\hline$r$ & \multicolumn{1}{c}{$e_{r}$} & $c \delta(r)$ \\
\hline 1.1 & 3.596 & 12.64 \\
1.2 & 1.507 & 3.318 \\
1.3 & .8045 & 1.467 \\
1.4 & .4851 & .7990 \\
1.6 & .2171 & .3213 \\
2.0 & $.6747(-1)$ & $.9112(-1)$ \\
3.0 & $.1047(-1)$ & $.1315(-1)$ \\
4.0 & $.3062(-2)$ & $.3755(-2)$ \\
6.0 & $.5726(-3)$ & $.6908(-3)$ \\
8.0 & $.1778(-3)$ & $.2133(-3)$ \\
\hline
\end{tabular}

A similar formula can be established for cross-product cubature rules.

Inequalities (2.2) and (2.6) suggest the error bound

$$
|E(f)| \leqq c \delta(r) M(r) .
$$

This error bound is more convenient than (2.2) in the sense that the error constant $c$, unlike $e_{r}$, does not depend on $r$. However, the bound given by (2.7) is somewhat worse than that given by (2.2). This is particularly true when $1<r<2$. Numerical experiments also indicate that $e_{r}$ is generally preferable to $c$ for characterizing (2.1) and comparing cubature rules.

Example 3. The Gauss-Legendre product rule

$$
\int_{-1}^{1} \int_{-1}^{1} f(x, y) d y d x=f\left( \pm \frac{3^{1 / 2}}{3}, \pm \frac{3^{1 / 2}}{3}\right)+E(f)
$$

has $c=.423280$ and $p=3$.

Since $E\left(x^{\mu} y^{\nu}\right) \geqq 0$ for $\mu+\nu \geqq 4$,

$$
e_{r}=\left(r \ln \frac{r+1}{r-1}\right)^{2}-4\left(1-\frac{3}{r^{2}}\right)^{-2},
$$

by (2.5). For this product rule,

$$
\delta(r)=\left(2 r^{4}-1\right) / r^{4}\left(r^{2}-1\right) .
$$

In Table 3, we have tabulated $e_{r}$ and $c \delta(r)$ to facilitate a comparison between the error bounds (2.2) and (2.7).

3. Barnhill and Chawla Error Bounds. Error estimates for (2.1) can be developed by using Hilbert space techniques. This theory is due to Barnhill [2] and is summarized in Stroud [19, Chapter 5]. Chawla [4] has recently obtained cubature error bounds similar to Barnhill's, through the use of Cauchy's integral formula. In this section, we compare (2.2) with the error bounds of Barnhill and Chawla.

Let $\xi_{\rho}$ denote the set of points in the complex plane which are interior to the ellipse with foci at $z= \pm 1$, semimajor axis $a$, semiminor axis $b=\left(a^{2}-1\right)^{1 / 2}$ and $\rho=(a+b)^{2}>1$. Designate by $\xi_{\rho} \times \xi_{\rho}$ the set of all pairs of complex numbers $(z, w)$, 
TABLE 4

Geometric Quantities for $\xi_{\rho}$

\begin{tabular}{ccrr}
\hline$a$ & $b$ & \multicolumn{1}{c}{$\rho$} & $a-b$ \\
\hline 1.6 & 1.2490 & 8.1168 & .3510 \\
2.0 & 1.7321 & 13.9282 & .2679 \\
3.0 & 2.8284 & 33.9706 & .1716 \\
4.0 & 3.8730 & 61.9839 & .1270 \\
6.0 & 5.9161 & 141.9930 & .0839 \\
8.0 & 7.9372 & 253.9961 & .0628 \\
\hline
\end{tabular}

where $z \in \xi_{\rho}$ in the complex $z$ plane and $w \in \xi_{\rho}$ in the complex $w$ plane. If $f(z, w)$ is analytic in the closed, elliptic bicylinder $\bar{\xi}_{\rho} \times \bar{\xi}_{\rho}$, then the theory in [2] and [4] can be used to bound $E(f)$ in (2.1).

Barnhill's error bound is given by

$$
|E(f)| \leqq d_{\rho}\|f\|
$$

where

$$
\|f\|^{2}=\iiint \int_{\xi_{\rho} \times \xi_{\rho}}|f(z, w)|^{2} d x d u d y d v
$$

and

$$
d_{\rho}^{2}=16 \pi^{-2} \sum_{\mu+\nu \geqq p+1} \frac{(\mu+1)(\nu+1)\left|E\left[U_{\mu}(x) U_{\nu}(y)\right]\right|^{2}}{\left(\rho^{\mu+1}-\rho^{-\mu-1}\right)\left(\rho^{\nu+1}-\rho^{-\nu-1}\right)} .
$$

In (3.3), $U_{\mu}(x)$ is the $\mu$ th Chebyshev polynomial of the second kind. As noted by Barnhill, the main difficulty in using (3.1) is the computation of $\|f\|$. Let

$$
M_{\rho}=\max _{\partial}|f(z, w)|,
$$

where $\partial=\left\{(z, w): z \in \partial \xi_{\rho}, w \in \partial \xi_{\rho}\right\}$ is the distinguished boundary of $\bar{\xi}_{\rho} \times \bar{\xi}_{\rho}$. By $(3.2)$,

$$
\|f\| \leqq \pi a b M_{\rho} .
$$

Combining (3.4) and (3.1), we obtain

$$
|E(f)| \leqq \pi a b d_{\rho} M_{\rho} .
$$

From the work of Chawla [4],

$$
|E(f)| \leqq c_{\rho} M_{\rho},
$$

where

$$
c_{\rho}^{2}=16 \sum_{\mu+\nu \geq p+1}^{\prime \prime} \rho^{-\mu-\nu}\left|E\left[T_{\mu}(x) T_{\nu}(y)\right]\right|^{2} .
$$

(It should be noted that $\rho=(a+b)^{2}$ here, whereas $\rho=(a+b)$ in [4].) The double prime on the summation sign indicates that the terms having $\mu=0$ or $\nu=0$ are to be multiplied by $\frac{1}{4}$. (Due to a misprint, the previous multiplier is given incorrectly as $\frac{1}{2}$ in [4].) In (3.7), $T_{\mu}(x)$ denotes the $\mu$ th Chebyshev polynomial of the first kind. 
Suppose $f \in A\left(B_{a}\right)$. Since $b=\frac{1}{2}\left(\rho^{1 / 2}-\rho^{-1 / 2}\right)<\frac{1}{2}\left(\rho^{1 / 2}+\rho^{-1 / 2}\right)=a, \bar{\xi}_{\rho} \times \bar{\xi}_{\rho} \subseteq B_{a}$ and $M_{\rho} \leqq M(a)$. By (3.5) and (3.6),

$$
|E(f)| \leqq \pi a b d_{\rho} M(a)
$$

and

$$
|E(f)| \leqq c_{\rho} M(a) .
$$

Table 4 indicates that $a \approx b$ for large $\rho$. More precisely, $a-b=\rho^{-1 / 2}$. For large $\rho$, the error bounds (3.8) and (3.9) are more convenient and give essentially the same results as (3.5) and (3.6), respectively.

If we take $r=a$ in (2.2), then (2.2), (3.8), and (3.9) have analogous forms. For large $\rho$, it is reasonable to compare these error bounds by comparing $e_{a}$ with $\pi a b d_{\rho}$ and $c_{\rho}$.

Let

$$
\begin{aligned}
\Sigma_{a} & =\sum_{\mu+\nu=p+1}\left|E\left(x^{\mu} y^{\nu}\right)\right|, \\
\Sigma_{d} & =\left[\sum_{\mu+\nu=p+1}(\mu+1)(\nu+1)\left|E\left(x^{\mu} y^{\nu}\right)\right|^{2}\right]^{1 / 2},
\end{aligned}
$$

and

$$
\Sigma_{c}=\left[\sum_{\mu+\nu=p+1}\left|E\left(x^{\mu} y^{\nu}\right)\right|^{2}\right]^{1 / 2} .
$$

THEOREM 2. Let $r=a=\left(\rho^{1 / 2}+\rho^{-1 / 2}\right) / 2$ in (2.3). Then

$$
\lim _{\rho \rightarrow \infty} e_{a} / \pi a b d_{\rho}=\Sigma_{a} / \Sigma_{d}
$$

and

$$
\lim _{\rho \rightarrow \infty} e_{a} / c_{\rho}=\Sigma_{a} / \Sigma_{c} .
$$

Proof. For each of the representations (2.3), (3.3) and (3.7), consider the first sum on the right-hand side of the decomposition

$$
\sum_{\mu+\nu \geq p+1}(\cdot)=\sum_{\mu+\nu=p+1}(\cdot)+\sum_{\mu+\nu \geq p+2}(\cdot) \text {. }
$$

Since $a=\rho^{1 / 2}\left(1+\rho^{-1}\right) / 2$,

$$
\sum_{\mu+\nu=p+1} a^{-\mu-\nu}\left|E\left(x^{\mu} y^{\nu}\right)\right| \leqq 2^{(p+1)} \rho^{-(p+1) / 2}\left(1+\rho^{-1}\right)^{-(p+1)} \Sigma_{a} .
$$

$E\left[U_{\mu}(x) U_{\nu}(y)\right]=2^{p+1} E\left(x^{\mu} y^{\nu}\right)$ for $\mu+\nu=p+1$, and it follows that

$$
\begin{aligned}
16 \pi^{-2} \sum_{\mu+\nu=p+1} \frac{(\mu+1)(\nu+1)\left|E\left[U_{\mu}(x) U_{\nu}(y)\right]\right|^{2}}{\left(\rho^{\mu+1}-\rho^{-\mu-1}\right)\left(\rho^{\nu+1}-\rho^{-\nu-1}\right)} \\
=2^{2 p+6} \rho^{-(p+3)} \pi^{-2} \sum_{\mu+\nu=p+1} \frac{(\mu+1)(\nu+1)\left|E\left(x^{\mu} y^{\nu}\right)\right|^{2}}{\left(1-\rho^{-2 \mu-2}\right)\left(1-\rho^{-2 \nu-2}\right)} .
\end{aligned}
$$

For $\mu+\nu=p+1$ and $\mu, \nu \neq 0$, we have $E\left[T_{\mu}(x) T_{\nu}(y)\right]=2^{p-1} E\left(x^{\mu} y^{\nu}\right)$. In addition, $E\left[T_{p+1}(x)\right]=2^{p} E\left(x^{p+1}\right)$ and $E\left[T_{p+1}(y)\right]=2^{p} E\left(y^{p+1}\right)$. Thus, 


$$
16 \sum_{\mu+\nu=p+1}^{\prime \prime} \rho^{-\mu-\nu}\left|E\left[T_{\mu}(x) T_{\nu}(y)\right]\right|^{2}=2^{2 p+2} \rho^{-(p+1)} \Sigma_{c}^{2} .
$$

By (2.3) and (3.12),

$$
e_{a} \sim 2^{p+1} \rho^{-(p+1) / 2} \Sigma_{a} .
$$

Since $a b=\left(\rho-\rho^{-1}\right) / 4,(3.3)$ and (3.13) imply

$$
\pi a b d_{\rho} \sim 2^{p+1} \rho^{-(p+1) / 2} \Sigma_{d} .
$$

Finally, (3.7) and (3.14) give

$$
c_{\rho} \sim 2^{p+1} \rho^{-(p+1) / 2} \Sigma_{c} .
$$

The proof follows from these asymptotic equalities.

It is possible to simplify the right-hand sides of (3.10) and (3.11) if (2.1) is a crossproduct rule. Let the two quadrature rules

$$
\int_{-1}^{1} \omega_{1}(x) f_{1}(x) d x \approx \sum_{i=1}^{N_{1}} w_{1, i} f_{1}\left(x_{i}\right)
$$

and

$$
\int_{-1}^{1} \omega_{2}(y) f_{2}(y) d y \approx \sum_{i=1}^{N_{2}} w_{2, i} f_{2}\left(y_{i}\right)
$$

have precision $p_{1}$ and $p_{2}$, respectively. The cross-product rule generated by these rules,

$$
\int_{-1}^{1} \int_{-1}^{1} \omega_{1}(x) \omega_{2}(y) f(x, y) d y d x=\sum_{i=1}^{N_{1}} \sum_{i=1}^{N_{2}} w_{1, i} w_{2, i} f\left(x_{i}, y_{i}\right)+E(f),
$$

has precision $p=\min \left\{p_{1}, p_{2}\right\}$.

Corollary 1. If $p_{1} \neq p_{2}$, then

$$
\lim _{\rho \rightarrow \infty} e_{a} / \pi a b d_{\rho}=(p+2)^{-1 / 2} \text { and } \lim _{\rho \rightarrow \infty} e_{a} / c_{\rho}=1 .
$$

If $p_{1}=p_{2}$ and $E\left(x^{p+1}\right)=E\left(y^{p+1}\right)$ in (3.15), then

$$
\lim _{\rho \rightarrow \infty} \frac{e_{a}}{\pi a b d_{\rho}}=\left[\frac{2}{p+2}\right]^{1 / 2} \text { and } \lim _{\rho \rightarrow \infty} \frac{e_{a}}{c_{\rho}}=2^{1 / 2} .
$$

Proof. Suppose $p_{1} \neq p_{2}$. With no loss of generality, we may assume $p=p_{1}$. All of the errors $E\left(x^{\mu} y^{\nu}\right), \mu+\nu=p+1$, are zero except $E\left(x^{p+1}\right)$. Thus, $\Sigma_{a}=\left|E\left(x^{p+1}\right)\right|$, $\Sigma_{d}=(p+2)^{1 / 2}\left|E\left(x^{p+1}\right)\right|$ and $\Sigma_{c}=\left|E\left(x^{p+1}\right)\right|$.

If $p_{1}=p_{2}=p$ and $E\left(x^{p+1}\right)=E\left(y^{\nu+1}\right)$, then $\Sigma_{a}=2\left|E\left(x^{p+1}\right)\right|, \Sigma_{d}=[2(p+2)]^{1 / 2}$. $\left|E\left(x^{p+1}\right)\right|$ and $\Sigma_{c}=\sqrt{ } 2\left|E\left(x^{p+1}\right)\right|$, since $E\left(x^{p+1}\right)$ and $E\left(y^{p+1}\right)$ are the only nonzero errors of the form $E\left(x^{\mu} y^{\nu}\right), \mu+\nu=p+1$. The proof now follows from Theorem 2.

We mention that the second part of the corollary applies to cross-product rules in which the same quadrature rule is used in both the $x$ and $y$ variables.

It is possible to bracket the right-hand sides of (3.10) and (3.11) for nonproduct cubature rules. Let $n$ denote the number of nonzero terms in the set of errors $\left\{E\left(x^{\mu} y^{\nu}\right): \mu+\nu=p+1\right\}$.

COROLlary 2. 


$$
\frac{2}{p+3} \leqq \lim _{\rho \rightarrow \infty} \frac{e_{a}}{\pi a b d_{\rho}} \leqq\left[\frac{n}{p+2}\right]^{1 / 2}
$$

and

$$
1 \leqq \lim _{\rho \rightarrow \infty} \frac{e_{a}}{c_{\rho}} \leqq n^{1 / 2}
$$

Proof. The proof follows from Theorem 2 by using the relations

$$
\begin{aligned}
& \min _{\mu+\nu=p+1}(\mu+1)(\nu+1)=p+2, \\
& \max _{\mu+\nu=p+1}(\mu+1)(\nu+1) \leqq(p+3)^{2} / 4
\end{aligned}
$$

and

$$
\Sigma_{c} \leqq \Sigma_{a} \leqq n^{1 / 2} \Sigma_{c}
$$

to obtain upper and lower bounds for $\Sigma_{a} / \Sigma_{d}$ and $\Sigma_{a} / \Sigma_{c}$.

Note that $n \leqq p+2$ and, therefore, the right-hand side of (3.16) is at most 1 . For fully symmetric rules, $n \leqq(p+3) / 2$. We remark that it is possible to have equality on the left- or right-hand sides of (3.16) or (3.17). It is not hard to construct specific rules to exhibit this. In fact, Corollary 1 defines classes of rules for which we have equality on the right-hand side of (3.16) and both sides of (3.17).

Example 4. The fully symmetric rule

$$
\begin{aligned}
\int_{-1}^{1} \int_{-1}^{1} f(x, y) d y d x= & \frac{40}{49}\left[f\left( \pm \frac{105^{1 / 2}}{15}, 0\right)+f\left(0, \pm \frac{105^{1 / 2}}{15}\right)\right] \\
& +\frac{9}{49}\left[f\left( \pm \frac{7^{1 / 2}}{3}, \pm \frac{7^{1 / 2}}{3}\right)\right]+E(f)
\end{aligned}
$$

has $p=5$. The Barnhill error constants $d_{\rho}$ for this rule are given in [19, p. 249]. The Chawla error constants $c_{\rho}$ can be computed from (3.7).

For this rule,

$$
\begin{aligned}
e_{a} & \sim 17.7710 \rho^{-3} \\
\pi a b d_{\rho} & \sim 31.1827 \rho^{-3}
\end{aligned}
$$

and

$$
c_{\rho} \sim 8.9700 \rho^{-3} \text {. }
$$

These quantities are tabulated in Table 5.

TABLE 5

Error Constants for Example 4

\begin{tabular}{crcccccc}
\hline \multicolumn{1}{c}{$a$} & \multicolumn{1}{c}{$\rho$} & $e_{a}$ & $17.7710 \rho^{-3}$ & $\pi a b d_{\rho}$ & $31.1827 \rho^{-3}$ & $c_{\rho}$ & $8.9700 \rho^{-3}$ \\
\hline 1.6 & 8.1168 & $.3549(-1)$ & $.3323(-1)$ & $.5765(-1)$ & $.5831(-1)$ & $.1714(-1)$ & $.1677(-1)$ \\
2.0 & 13.9282 & $.6860(-2)$ & $.6577(-2)$ & $.1149(-1)$ & $.1154(-1)$ & $.3344(-2)$ & $.3320(-2)$ \\
3.0 & 33.9706 & $.4618(-3)$ & $.4533(-3)$ & $.7749(-3)$ & $.7954(-3)$ & $.2291(-3)$ & $.2288(-3)$ \\
4.0 & 61.9839 & $.7540(-4)$ & $.7462(-4)$ & $.1309(-3)$ & $.1309(-3)$ & $.3767(-4)$ & $.3767(-4)$ \\
6.0 & 141.9930 & $.6236(-5)$ & $.6207(-5)$ & $.1089(-4)$ & $.1089(-4)$ & $.3133(-5)$ & $.3133(-5)$ \\
8.0 & 253.9961 & $.1087(-5)$ & $.1084(-5)$ & $.1903(-5)$ & $.1903(-5)$ & $.5474(-6)$ & $.5474(-6)$ \\
\hline
\end{tabular}


In this example, $n=4$ and Corollary 2 gives

$$
.2500 \leqq \lim _{\rho \rightarrow \infty} e_{a} / \pi a b d_{\rho} \leqq .7560
$$

and

$$
1 \leqq \lim _{\rho \rightarrow \infty} e_{a} / c_{\rho} \leqq 2 .
$$

The actual limits are equal to .5699 and 1.9812 , to 4 places.

4. Error Constants. The constant $e_{r}$ gives valuable information about (2.1), since it allows one to compare the relative merits of one cubature rule with another.

TABLE 6

\begin{tabular}{|c|c|c|c|c|}
\hline$r$ & $\begin{array}{l}C_{2}: 5-1 \\
c=.2045 \\
e_{r}\end{array}$ & $\begin{array}{l}C_{2}: 5-4 \\
c=.2044 \\
e_{r}\end{array}$ & $\begin{array}{l}C_{n}: 5-5, n=2 \\
c=.4444 \\
e_{r}\end{array}$ & $\begin{array}{l}C_{2}: 5-5 \\
c=.6222 \\
e_{r}\end{array}$ \\
\hline 1.1 & 1.789 & 1.650 & 9.290 & 10.27 \\
\hline 1.2 & .5435 & .4754 & 1.745 & 2.039 \\
\hline 1.3 & .2252 & .1911 & .5882 & .7131 \\
\hline 1.4 & .1098 & $.9131(-1)$ & .2554 & .3179 \\
\hline 1.6 & $.3467(-1)$ & $.2813(-1)$ & $.7110(-1)$ & $.9166(-1)$ \\
\hline 2.0 & $.6347(-2)$ & $.5035(-2)$ & $.1171(-1)$ & $.1566(-1)$ \\
\hline 3.0 & $.4078(-3)$ & $3180(-3)$ & $.6968(-3)$ & $.9626(-3)$ \\
\hline 4.0 & $.6558(-4)$ & $.5089(-4)$ & $.1096(-3)$ & $.1530(-3)$ \\
\hline 6.0 & $.5367(-5)$ & $.4151(-5)$ & $.8839(-5)$ & $.1243(-4)$ \\
\hline 8.0 & $.9324(-6)$ & $.7203(-6)$ & $.1528(-5)$ & $.2154(-5)$ \\
\hline
\end{tabular}

Values of $e_{r}, c$ for $D:-1 \leqq x, y \leqq 1$

TABLE $6^{\prime}$

\begin{tabular}{|c|c|c|c|c|}
\hline rule & $\begin{array}{l}C_{2}: 5-6 \\
c=.6222 \\
e_{r}\end{array}$ & $\begin{array}{l}C_{2}: 7-1 \\
c=.1367 \\
e_{r}\end{array}$ & $\begin{array}{l}C_{2}: 7-3 \\
c=.5873(-1) \\
e_{r}\end{array}$ & $\begin{array}{l}C_{2}: 7-5 \\
c=.4423 \\
e_{r}\end{array}$ \\
\hline 1.1 & 6.432 & .7911 & .4688 & 4.131 \\
\hline 1.2 & 1.246 & .1668 & $.9721(-1)$ & .6689 \\
\hline 1.3 & .4254 & $.5309(-1)$ & $3200(-1)$ & .1941 \\
\hline 1.4 & .1858 & $.2095(-1)$ & $.1337(-1)$ & $.7301(-1)$ \\
\hline 1.6 & $.5192(-1)$ & $.4726(-2)$ & $.3057(-2)$ & $.1563(-1)$ \\
\hline 2.0 & $.8550(-2)$ & $.5268(-3)$ & $.3477(-3)$ & $.1649(-2)$ \\
\hline 3.0 & $.5068(-3)$ & $.1474(-4)$ & $.9622(-5)$ & $.4357(-4)$ \\
\hline 4.0 & $.7955(-4)$ & $.1332(-5)$ & $.8602(-6)$ & $.3852(-5)$ \\
\hline 6.0 & $.6405(-5)$ & $4851(-7)$ & $.3102(-7)$ & $.1380(-6)$ \\
\hline 8.0 & $.1107(-5)$ & $4745(-8)$ & $.3022(-8)$ & $.1341(-7)$ \\
\hline
\end{tabular}

Values of $e_{r}, c$ for $D:-1 \leqq x, y \leqq 1$ 
TABLE 7

Values of $e_{r}, c$ for $D: x^{2}+y^{2} \leqq 1$

\begin{tabular}{lllll}
\hline rule & $\begin{array}{l}S_{2}: 5-2 \\
c=.7977(-1)\end{array}$ & $\begin{array}{l}S_{n}: 5-5, n=2 \\
c=.6124(-1) \\
e_{r}\end{array}$ & $\begin{array}{l}S_{n}: 5-6, n=2 \\
e_{r}=.5223(-1)\end{array}$ & $\begin{array}{l}S_{2}: 7-1 \\
c=.2431(-1) \\
e_{r}\end{array}$ \\
\hline 1.1 & .4361 & .3164 & .2563 & .1177 \\
1.2 & .1661 & .1102 & $.8350(-1)$ & $.3234(-1)$ \\
1.3 & $.7832(-1)$ & $.4908(-1)$ & $.3555(-1)$ & $.1240(-1)$ \\
1.4 & $.4162(-1)$ & $.2505(-1)$ & $.1757(-1)$ & $.5646(-2)$ \\
1.6 & $.1464(-1)$ & $.8366(-2)$ & $.5613(-2)$ & $.1558(-2)$ \\
2.0 & $.2988(-2)$ & $.1618(-2)$ & $.1035(-2)$ & $.2172(-3)$ \\
3.0 & $.2107(-3)$ & $.1089(-3)$ & $.6664(-4)$ & $.7442(-5)$ \\
4.0 & $.3492(-4)$ & $.1778(-4)$ & $.1072(-4)$ & $.7187(-6)$ \\
6.0 & $.2917(-5)$ & $.1470(-5)$ & $.8771(-6)$ & $.2440(-7)$ \\
8.0 & $.5103(-6)$ & $.2563(-6)$ & $.1524(-6)$ & $.2722(-8)$ \\
\hline
\end{tabular}

TABLE 8

Values of $e_{r}, c$ for $D: x+y \leqq 1, x, y \geqq 0$

\begin{tabular}{llll}
\hline & $T_{n}: 3-1, n=2$ & $\begin{array}{c}\text { Conical Product } \\
\text { 4-point rule [20] } \\
c=.4969(-2)\end{array}$ & $\begin{array}{l}T_{n}: 3-8, n=2 \\
c=.2500(-1) \\
e_{r}\end{array}$ \\
\hline $\begin{array}{l}c=.6779(-2) \\
e_{r}\end{array}$ & $.5476(-1)$ & .2598 \\
1.1 & $.6838(-1)$ & $.2412(-1)$ & $.8166(-1)$ \\
1.2 & $.3164(-1)$ & $.1274(-1)$ & $.3720(-1)$ \\
1.3 & $.1728(-1)$ & $.7484(-2)$ & $.2011(-1)$ \\
1.4 & $.1040(-1)$ & $.3151(-2)$ & $.7735(-2)$ \\
1.6 & $.4531(-2)$ & $.8753(-3)$ & $.1993(-2)$ \\
2.0 & $.1309(-2)$ & $.1128(-3)$ & $.2432(-3)$ \\
3.0 & $.1759(-3)$ & $.2967(-4)$ & $.6289(-4)$ \\
4.0 & $.4704(-4)$ & $.4941(-5)$ & $.1035(-4)$ \\
6.0 & $.7952(-5)$ & $.1443(-5)$ & $.3006(-5)$ \\
8.0 & $.2337(-5)$ & & \\
\hline
\end{tabular}

TABLE 9

Values of $c$ for (4.1) and (4.2)

\begin{tabular}{ccc}
\hline & rule (4.1) & rule (4.2) \\
$n$ & $c$ & $c$ \\
\hline 2 & .1963 & .1963 \\
3 & $.7977(-1)$ & $.7977(-1)$ \\
4 & $.3597(-1)$ & $.3931(-1)$ \\
5 & $.2042(-1)$ & $.2237(-1)$ \\
6 & $.1216(-1)$ & $.1396(-1)$ \\
7 & $.8083(-2)$ & $.9289(-2)$ \\
\hline
\end{tabular}


TABLE 10

Values of $\delta(r)$ for the Rules in Tables 6-8

\begin{tabular}{lccccc}
\hline rule & & & & & \\
$r$ & Group A & Group B & Group C & $C_{2}: 5-1$ & $C_{2}: 5-4$ \\
\hline 1.1 & 28.4976 & 26.2397 & 112.6972 & 27.9331 & 26.9022 \\
1.2 & 6.8751 & 5.5355 & 28.9352 & 6.5402 & 5.9727 \\
1.3 & 2.7651 & 1.9364 & 12.6435 & 2.5579 & 2.2282 \\
1.4 & 1.3671 & .8358 & 6.8331 & 1.2343 & 1.0337 \\
1.6 & .4540 & .2155 & 2.7127 & .3944 & .3315 \\
2.0 & $.9028(-1)$ & $.2778(-1)$ & .7500 & $.7466(-1)$ & $.5512(-1)$ \\
3.0 & $.6366(-2)$ & $.8788(-3)$ & .1018 & $.4994(-2)$ & $.3470(-2)$ \\
4.0 & $.1059(-2)$ & $.8246(-4)$ & $.2778(-1)$ & $.8148(-3)$ & $.5555(-3)$ \\
6.0 & $.8881(-4)$ & $.3079(-5)$ & $.4815(-2)$ & $.6738(-4)$ & $.4535(-4)$ \\
8.0 & $.1556(-4)$ & $.3037(-6)$ & $.1435(-2)$ & $.1174(-4)$ & $.7874(-5)$ \\
\hline
\end{tabular}

Stroud [19] has recently compiled an extensive list of cubature rules. Each rule that appears in [19, Chapter 8] has been assigned a unique symbol such as $C_{2}: 7-3$. Here, $C_{2}$ denotes the square $[-1,1] \times[-1,1]$; the 7 designates the precision of the rule; and the 3 means that this is the third rule of precision 7 given for $C_{2}$ in Stroud's listing. In Tables 6, 6', 7 and 8, we have used Stroud's notation to define a cubature rule rather than listing its weights and nodes as in (2.1).

Table 9 gives the error coefficient $c$ for two families of cubature rules for the disc, $x^{2}+y^{2} \leqq 1$. The spherical product rules [20] are defined by

$$
\iint_{x^{2}+y^{2} \leqq 1} f(x, y) d x d y \approx \frac{\pi}{n} \sum_{\mu=1}^{n} \sum_{\nu=1}^{n} A_{\mu} f\left(a_{\mu} \sin \frac{2 \nu-1}{2 n} \pi, a_{\mu} \cos \frac{2 \nu-1}{2 n} \pi\right),
$$

where the $A_{\mu}, a_{\mu}$ are the weights and abscissas for the $n$-point Gaussian quadrature rule with weight function $|x|$ on $[-1,1]$. The generalized product rules [12] are given by

$$
\begin{aligned}
& \iint_{x^{2}+y^{2} \leqq 1} f(x, y) d x d y \\
& \quad \approx \frac{\pi}{n+1} \sum_{\mu=1}^{n} \sum_{\nu=1}^{n} B_{\nu} \sin ^{2} \frac{\mu \pi}{n+1} f\left(\cos \frac{\mu \pi}{n+1}, b_{\nu} \sin \frac{\mu \pi}{n+1}\right),
\end{aligned}
$$

where the $B_{v}, b_{v}$ are the weights and abscissas for the $n$-point Gauss-Legendre rule. Both (4.1) and (4.2) have precision $2 n-1$.

In carrying out the calculations in this paper, it was observed that the errors $E\left(x^{\mu} y^{\nu}\right), \mu+\nu \geqq p+1$, do not change sign for the following nonproduct rules: $C_{n}: 5-5, n=2 ; C_{2}: 5-5, C_{2}: 5-6, C_{2}: 7-5, S_{n}: 5-5, n=2 ; S_{n}: 5-6, n=2$ and the rule in Example 2. Consequently, (2.4) holds for these cubature rules.

In Table 10, we give values of $\delta(r)$ for the rules in Tables 6-8. It is convenient to group some of these rules as follows:

Group A: $C_{n}: 5-5, n=2, C_{2}: 5-5, C_{2}: 5-6, S_{2}: 5-2, S_{n}: 5-5, n=2, S_{n}: 5-6$, $n=2$.

Group B: $C_{2}: 7-1, C_{2}: 7-3, C_{2}: 7-5, S_{2}: 7-1$.

Group C: The rules in Table 8. 
5. Acknowledgments. The author would like to thank the referee of this paper for making some helpful comments. Appreciation is also expressed to Professors R. E. Barnhill and F. Stenger for reading a preliminary version of this paper.

6. Note Added in Proof. It has been pointed out to the author by one of the editors, that the double integral in illustrative Example 2 has the closed-form value of $\pi\left(16-254^{1 / 2}\right)$.

\section{Department of Mathematics}

University of Georgia

Athens, Georgia 30601

1. A. C. Ahlin, "On error bounds for Gaussian cubature," SIAM Rev., v. 4, 1962, pp. 25-39. MR 27 \#2105.

2. (a) R. E. BARNHILL, "An error analysis for numerical multiple integration. I and II," Math. Comp., v. 22, 1968, pp. 98-109, 286-292. MR 37 \#2438; \#6029.

(b) R. E. BARNHILl \& G. M. Nielson, "An error analysis for numerical multiple integration. III," Math. Comp., v. 24, 1970, pp. 301-314. MR 43 \#1418.

3. M. M. CHAWLA, "On the estimation of errors of Gaussian cubature formulas," SIAM J. Numer. Anal., v. 5, 1968, pp. 172-181. MR 36 \#324.

4. M. M. ChaWLA, "Estimating errors of numerical approximation for analytic functions," Numer. Math., v. 16, 1971, pp. 370-374. MR 44 \#1186.

5. B. A. Fuks, Theory of Analytic Functions of Several Complex Variables, Transl. Math. Monographs, vol. 8, Amer. Math. Soc., Providence, R.I., 1963. MR 29 \#6049.

6. S. HABER, "Numerical evaluation of multiple integrals," SIAM Rev., v. 12, 1970, pp. 481-526. MR 44 \#2342.

7. P. C. HAMMER \& A. H. Stroud, "Numerical evaluation of multiple integrals. II," $M T A C$, v.12, 1958, pp. 272-280. MR 21 \#970.

8. F. G. LeTHER, "Cross-product cubature error bounds," Math. Comp., v. 24, 1970, pp. 583-592. MR 43 \#1426.

9. F. G. LETHER, "An error representation for product cubature rules," SIAM J. Numer. Anal., v. 7, 1970, pp. 363-365. MR 42 \#2659.

10. F. G. LETHER, "Error bounds for fully symmetric cubature rules," SIAM J. Numer. Anal., v. 8, 1971, pp. 49-60. MR 43 \#7064.

11. F. G. LETHER, "Cubature error bounds for Gauss-Legendre product rules," SIAM J. Numer. Anal., v. 8, 1971, pp. 36-42. MR 43 \#7063.

12. F. G. LETHER, "A generalized product rule for the circle," SIAM J. Numer. Anal., v. 8, 1971, pp. 249-253. MR 44 \#2343.

13. S. M. NIKol'SkIĬ, Quadrature Formulas, Fizmatgiz, Moscow, 1958. (Russian)

14. A. SARD, Linear Approximation, Math. Surveys, No. 9, Amer. Math. Soc., Providence, R.I., 1963. MR 28 \#1429.

15. V. L. N. SARMa and A. H. Stroud, "Eberlein measure and mechanical quadrature formula. II. Numerical results," Math. Comp., v. 23, 1969, pp, 781-784. MR 41 \#2929.

16. D. D. Stancu, "The remainder of certain linear approximation formulas in two variables," J. Soc. Indust. Appl. Math. Ser. B. Numer. Anal., v. 1, 1964, pp. 137-163. MR 31 \#1503.

17. F. Stenger, "Bounds on the error of Gauss-type quadrature," Numer. Math., v. 8, 1966, pp. 150-160. MR 33 \#5120.

18. F. STENGER, "Error bounds for the evaluation of integrals by repeated Gauss-type formulae," Numer. Math., v. 9, 1966, pp. 200-213. MR 34 \#5289.

19. A. H. STroud, Approximate Calculation of Multiple Integrals, Prentice-Hall, Englewood Cliffs, N.J., 1971.

20. A. H. STroud \& D. SeCrest, Gaussian Quadrature Formulas, Prentice-Hall, Englewood Cliffs, N.J., 1966. MR 34 \#2185. 